Internet Engineering Task Force (IETF)

J. Klensin

Request for Comments: 5890

August 2010

Obsoletes: 3490

Category: Standards Track

ISSN : $2070-1721$

\title{
Internationalized Domain Names for Applications (IDNA): Definitions and Document Framework
}

\section{Abstract}

This document is one of a collection that, together, describe the protocol and usage context for a revision of Internationalized Domain Names for Applications (IDNA), superseding the earlier version. It describes the document collection and provides definitions and other material that are common to the set.

Status of This Memo

This is an Internet Standards Track document.

This document is a product of the Internet Engineering Task Force (IETF). It represents the consensus of the IETF community. It has received public review and has been approved for publication by the Internet Engineering Steering Group (IESG). Further information on Internet Standards is available in section 2 of RFC 5741.

Information about the current status of this document, any errata, and how to provide feedback on it may be obtained at http://www.rfc-editor.org/info/rfc5890. 
Copyright Notice

Copyright (c) 2010 IETF Trust and the persons identified as the document authors. All rights reserved.

This document is subject to BCP 78 and the IETF Trust's Legal Provisions Relating to IETF Documents

(http://trustee.ietf.org/license-info) in effect on the date of publication of this document. Please review these documents carefully, as they describe your rights and restrictions with respect to this document. Code Components extracted from this document must include Simplified BSD License text as described in section $4 . e$ of the Trust Legal Provisions and are provided without warranty as described in the Simplified BSD License.

This document may contain material from IETF Documents or IETF Contributions published or made publicly available before November 10, 2008. The person(s) controlling the copyright in some of this material may not have granted the IETF Trust the right to allow modifications of such material outside the IETF Standards Process. Without obtaining an adequate license from the person(s) controlling the copyright in such materials, this document may not be modified outside the IETF standards Process, and derivative works of it may not be created outside the IETF Standards Process, except to format it for publication as an RFC or to translate it into languages other than English. 
Table of Contents

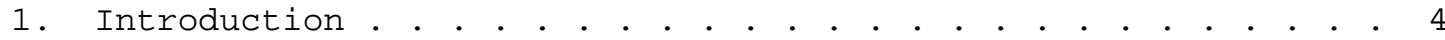

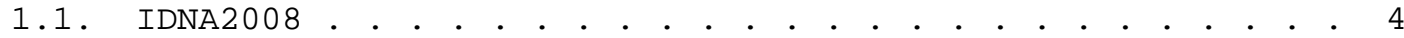

1.1.1. Audiences . . . . . . . . . . . . . . . . . . . . 4

1.1.2. Normative Language . . . . . . . . . . . . . . 5

1.2. Road Map of IDNA2008 Documents . . . . . . . . . . . . . . 5

2. Definitions and Terminology . • . • . . . . . . • . . . . . . 6

2.1. Characters and Character Sets . . . . . . . . . . . . 6

2.2. DNS-Related Terminology . . . . . . . . . . . . . . . . 6

2.3. Terminology Specific to IDNA . . . . . . . . . . . . . . . 7

2.3.1. LDH Label . . . . . . . . . . . . . . . . . . . 7

2.3.2. Terms for IDN Label Codings . . . . . . . . . . . . . 11

2.3.2.1. IDNA-valid strings, A-label, and U-label. . . . . 11

2.3.2.2. NR-LDH Label . . . . . . . . . . . . . . 13

2.3.2.3. Internationalized Domain Name and

Internationalized Label . . . . . . . . . . 13

2.3.2.4. Label Equivalence . . . . . . . . . . . . . . 14

2.3.2.5. ACE Prefix . . . . . . . . . . . . . . . . 14

2.3.2.6. Domain Name Slot. . . . . . . . . . . . . . 14

2.3.3. Order of Characters in Labels . . . . . . . . . . . 15

2.3.4. Punycode is an Algorithm, Not a Name or Adjective . 15

3. IANA Considerations . . . . . . . . . . . . . . . . . 16

4. Security Considerations . • • • • • . • . • . • . . . . . 16

4.1. General Issues . . . . . . • . . . . . . . . . . 16

4.2. U-label Lengths . . . . . . . . . . . . . . . . . 16

4.3. Local Character Set Issues . . . . . . . . . . . . . 17

4.4. Visually Similar Characters . . . . . . . . . . . . . . . 17

4.5. IDNA Lookup, Registration, and the Base DNS

Specifications . . . . . . . . . . . . . . . . . . . 18

4.6. Legacy IDN Label Strings . . . . . . . . . . . . . . . 18

4.7. Security Differences from IDNA2003 . . . . . . . . . . . 19

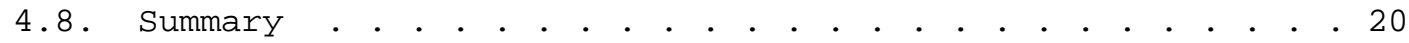

5. Acknowledgments . • . . . . . . . . . . . . . . . . 20

6. References... . . . . . . . . . . . . . . . . . . 20

6.1. Normative References . . . . . . . . . . . . . . . 20

6.2. Informative References . . . . . . . . . . . . . . . 21 


\section{Introduction}

\subsection{IDNA2008}

This document is one of a collection that, together, describe the protocol and usage context for a revision of Internationalized Domain Names for Applications (IDNA) that was largely completed in 2008, known within the series and elsewhere as "IDNA2008". The series replaces an earlier version of IDNA [RFC3490] [RFC3491]. For convenience, that version of IDNA is referred to in these documents as "IDNA2003". The newer version continues to use the Punycode algorithm [RFC3492] and ACE (ASCII-compatible encoding) prefix from that earlier version. The document collection is described in Section 1.2. As indicated there, this document provides definitions and other material that are common to the set.

\subsubsection{Audiences}

While many IETF specifications are directed exclusively to protocol implementers, the character of IDNA requires that it be understood and properly used by those whose responsibilities include making decisions about:

o what names are permitted in DNS zone files,

- policies related to names and naming, and

- the handling of domain name strings in files and systems, even with no immediate intention of looking them up.

This document and those documents concerned with the protocol definition, rules for handling strings that include characters written right to left, and the actual list of characters and categories will be of primary interest to protocol implementers. This document and the one containing explanatory material will be of primary interest to others, although they may have to fill in some details by reference to other documents in the set.

This document and the associated ones are written from the perspective of an IDNA-aware user, application, or implementation. While they may reiterate fundamental DNS rules and requirements for the convenience of the reader, they make no attempt to be comprehensive about DNS principles and should not be considered as a substitute for a thorough understanding of the DNS protocols and specifications. 


\subsubsection{Normative Language}

The key words "MUST", "MUST NOT", "REQUIRED", "SHALL", "SHALL NOT", "SHOULD", "SHOULD NOT", "RECOMMENDED", "MAY", and "OPTIONAL" in this document are to be interpreted as described in RFC 2119 [RFC2119].

\subsection{Road Map of IDNA2008 Documents}

IDNA2008 consists of the following documents:

- This document, containing definitions and other material that are needed for understanding other documents in the set. It is referred to informally in other documents in the set as "Defs" or "Definitions".

- A document, RFC 5894 [RFC5894], that provides an overview of the protocol and associated tables together with explanatory material and some rationale for the decisions that led to IDNA2008. That document also contains advice for registry operations and those who use Internationalized Domain Names (IDNs). It is referred to informally in other documents in the set as "Rationale". It is not normative.

- A document, RFC 5891 [RFC5891], that describes the core IDNA2008 protocol and its operations. In combination with the Bidi document, described immediately below, it explicitly updates and replaces $\mathrm{RFC} 3490$. It is referred to informally in other documents in the set as "Protocol".

- A document, RFC 5893 [RFC5893], that specifies special rules (Bidi) for labels that contain characters that are written from right to left.

- A specification, RFC 5892 [RFC5892], of the categories and rules that identify the code points allowed in a label written in native character form (defined more specifically as a "U-label" in Section 2.3.2.1 below), based on Unicode 5.2 [Unicode52] code point assignments and additional rules unique to IDNA2008. The Unicode-based rules are expected to be stable across Unicode updates and hence independent of Unicode versions. That specification obsoletes RFC 3941 and IDN use of the tables to which it refers. It is referred to informally in other documents in the set as "Tables". 
- A document [IDNA2008-Mapping] that discusses the issue of mapping characters into other characters and that provides guidance for doing so when that is appropriate. That document, referred to informally as "Mapping", provides advice; it is not a required part of IDNA.

2. Definitions and Terminology

\subsection{Characters and Character sets}

A code point is an integer value in the codespace of a coded character set. In Unicode, these are integers from 0 to 0x10FFFF.

Unicode [Unicode52] is a coded character set containing somewhat over 100,000 characters assigned to code points as of version 5.2. A single Unicode code point is denoted in these documents by "U+" followed by four to six hexadecimal digits, while a range of Unicode code points is denoted by two four to six digit hexadecimal numbers separated by "..", with no prefixes.

ASCII means US-ASCII [ASCII], a coded character set containing 128 characters associated with code points in the range $0000 \ldots 007 \mathrm{~F}$. Unicode is a superset of ASCII and may be thought of as a generalization of it; it includes all the ASCII characters and associates them with the equivalent code points.

"Letters" are, informally, generalizations from the ASCII and common-sense understanding of that term, i.e., characters that are used to write text and that are not digits, symbols, or punctuation. Formally, they are characters with a Unicode General Category value starting in "L" (see Section 4.5 of The Unicode Standard [Unicode52]).

\subsection{DNS-Related Terminology}

When discussing the DNS, this document generally assumes the terminology used in the DNS specifications [RFC1034] [RFC1035] as subsequently modified [RFC1123] [RFC2181]. The term "lookup" is used to describe the combination of operations performed by the IDNA2008 protocol and those actually performed by a DNS resolver. The process of placing an entry into the DNS is referred to as "registration". This is similar to common contemporary usage of that term in other contexts. Consequently, any DNS zone administration is described as a "registry", and the terms "registry" and "zone administrator" are used interchangeably, regardless of the actual administrative arrangements or level in the DNS tree. More details about that relationship are included in the Rationale document. 
The term "LDH code point" is defined in this document to refer to the code points associated with ASCII letters (Unicode code points 0041.005A and 0061.007A), digits (0030.0039), and the hyphen-minus (U+002D). "LDH" is an abbreviation for "letters, digits, hyphen" but is used specifically in this document to refer to the set of naming rules described in Section 2.3.1 below.

The base DNS specifications [RFC1034] [RFC1035] discuss "domain names" and "hostnames", but many people use the terms interchangeably, as do sections of these specifications. Lack of clarity about that terminology has contributed to confusion about intent in some cases. These documents generally use the term "domain name". When they refer to, e.g., hostname syntax restrictions, they explicitly cite the relevant defining documents. The remaining definitions in this subsection are essentially a review: if there is any perceived difference between those definitions and the definitions in the base DNS documents or those cited below, the definitions in the other documents take precedence.

A label is an individual component of a domain name. Labels are usually shown separated by dots; for example, the domain name "www.example.com" is composed of three labels: "www", "example", and "com". (The complete name convention using a trailing dot described in RFC 1123 [RFC1123], which can be explicit as in "www.example.com." or implicit as in "wWw.example.com", is not considered in this specification.) IDNA extends the set of usable characters in labels that are treated as text (as distinct from the binary string labels discussed in RFC 1035 and RFC 2181 [RFC2181] and bitstring ones [RFC2673]), but only in certain contexts. The different contexts for different sets of usable characters are outlined in the next section. For the rest of this document and in the related ones, the term "label" is shorthand for "text label", and "every label" means "every text label", including the expanded context.

\subsection{Terminology specific to IDNA}

This section defines some terminology to reduce dependence on terms and definitions that have been problematic in the past. The relationships among these definitions are illustrated in Figure 1 and Figure 2. In the first of those figures, the parenthesized numbers refer to the notes below the figure.

\subsubsection{LDH Label}

This is the classical label form used, albeit with some additional restrictions, in hostnames [RFC0952]. Its syntax is identical to that described as the "preferred name syntax" in section 3.5 of RFC 1034 [RFC1034] as modified by RFC 1123 [RFC1123]. Briefly, it is a 
string consisting of ASCII letters, digits, and the hyphen with the further restriction that the hyphen cannot appear at the beginning or end of the string. Like all DNS labels, its total length must not exceed 63 octets.

LDH labels include the specialized labels used by IDNA (described as "A-labels" below) and some additional restricted forms (also described below).

To facilitate clear description, two new subsets of LDH labels are created by the introduction of IDNA. These are called Reserved LDH labels (R-LDH labels) and Non-Reserved LDH labels (NR-LDH labels). Reserved LDH labels, known as "tagged domain names" in some other contexts, have the property that they contain "--" in the third and fourth characters but which otherwise conform to LDH label rules. Only a subset of the R-LDH labels can be used in IDNA-aware applications. That subset consists of the class of labels that begin with the prefix "xn--" (case independent), but otherwise conform to the rules for LDH labels. That subset is called "XN-labels" in this set of documents. XN-labels are further divided into those whose remaining characters (after the "xn--") are valid output of the Punycode algorithm [RFC3492] and those that are not (see below). The $\mathrm{XN}-\mathrm{labels}$ that are valid Punycode output are known as "A-labels" if they also meet the other criteria for IDNA-validity described below. Because LDH labels (and, indeed, any DNS label) must not be more than 63 octets in length, the portion of an XN-label derived from the Punycode algorithm is limited to no more than 59 ASCII characters. Non-Reserved LDH labels are the set of valid LDH labels that do not have "--" in the third and fourth positions.

A consequence of the restrictions on valid characters in the native Unicode character form (see U-labels) turns out to be that mixed-case annotation, of the sort outlined in Appendix A of RFC 3492 [RFC3492], is never useful. Therefore, since a valid A-label is the result of Punycode encoding of a U-label, A-labels should be produced only in lowercase, despite matching other (mixed-case or uppercase) potential labels in the DNS.

Some strings that are prefixed with "xn--" to form labels may not be the output of the Punycode algorithm, may fail the other tests outlined below, or may violate other IDNA restrictions and thus are also not valid IDNA labels. They are called "Fake A-labels" for convenience.

Labels within the class of R-LDH labels that are not prefixed with "xn--" are also not valid IDNA labels. To allow for future use of mechanisms similar to IDNA, those labels MUST NOT be processed as 


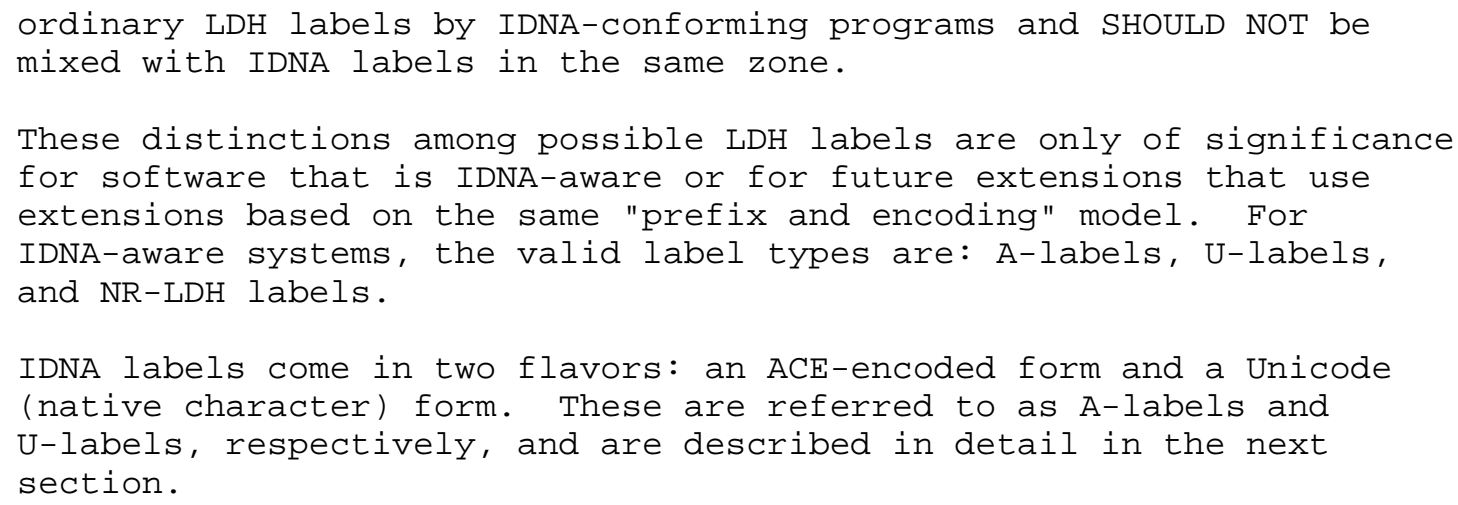


ASCII Label

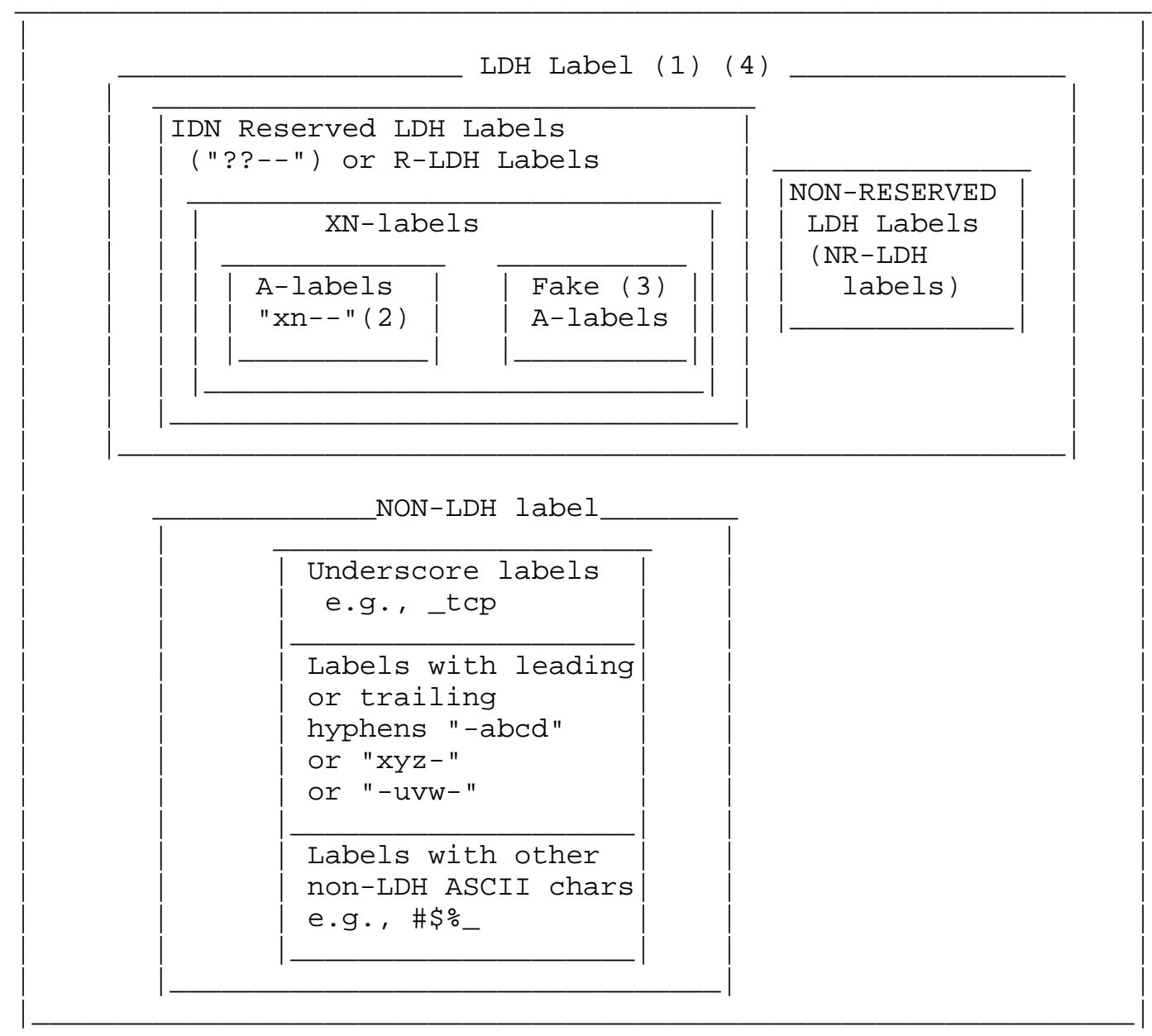

(1) ASCII letters (uppercase and lowercase), digits, hyphen. Hyphen may not appear in first or last position. No more than 63 octets.

(2) Note that the string following "xn--" must be the valid output of the Punycode algorithm and must be convertible into valid U-label form.

(3) Note that a Fake A-label has a prefix "xn--" but the remainder of the label is NOT the valid output of the Punycode algorithm.

(4) LDH label subtypes are indistinguishable to applications that are not IDNA-aware.

Figure 1: IDNA and Related DNS Terminology Space -- ASCII Labels 


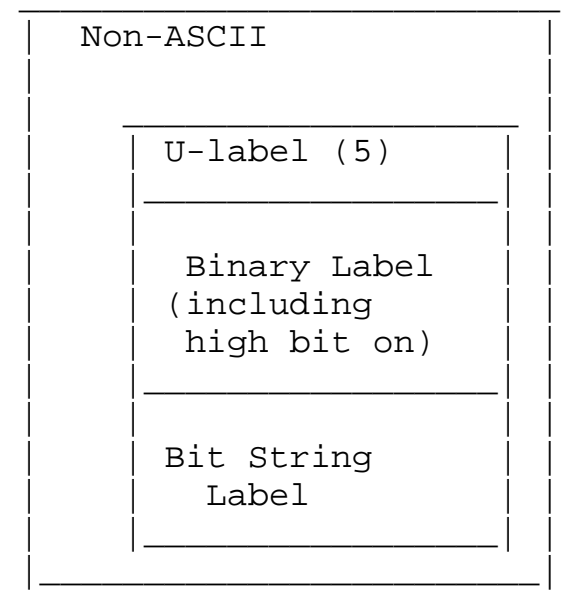

(5) To applications that are not IDNA-aware, U-labels are indistinguishable from Binary ones.

Figure 2: Non-ASCII Labels

\subsubsection{Terms for IDN Label Codings}

\subsubsection{IDNA-valid strings, A-label, and U-label}

For IDNA-aware applications, the three types of valid labels are "A-labels", "U-labels", and "NR-LDH labels", each of which is defined below. The relationships among them are illustrated in Figure 1 and Figure 2.

- A string is "IDNA-valid" if it meets all of the requirements of these specifications for an IDNA label. IDNA-valid strings may appear in either of the two forms defined immediately below, or may be drawn from the NR-LDH label subset. IDNA-valid strings must also conform to all basic DNS requirements for labels. These documents make specific reference to the form appropriate to any context in which the distinction is important.

- An "A-label" is the ASCII-Compatible Encoding (ACE, see Section 2.3.2.5) form of an IDNA-valid string. It must be a complete label: IDNA is defined for labels, not for parts of them and not for complete domain names. This means, by definition, that every A-label will begin with the IDNA ACE prefix, "xn--" (see Section 2.3.2.5), followed by a string that is a valid output of the Punycode algorithm [RFC3492] and hence a maximum of 59 ASCII characters in length. The prefix and string together must conform to all requirements for a label that can be stored in the 
DNS including conformance to the rules for LDH labels (Section 2.3.1). If and only if a string meeting the above requirements can be decoded into a U-label is it an A-label.

- A "U-label" is an IDNA-valid string of Unicode characters, in Normalization Form $\mathrm{C}$ (NFC) and including at least one non-ASCII character, expressed in a standard Unicode Encoding Form (such as $\mathrm{UTF}-8)$. It is also subject to the constraints about permitted characters that are specified in section 4.2 of the Protocol document and the rules in the sections 2 and 3 of the Tables document, the Bidi constraints in that document if it contains any character from scripts that are written right to left, and the symmetry constraint described immediately below. Conversions between U-labels and A-labels are performed according to the "Punycode" specification [RFC3492], adding or removing the ACE prefix as needed.

To be valid, U-labels and A-labels must obey an important symmetry constraint. While that constraint may be tested in any of several ways, an A-label A1 must be capable of being produced by conversion from a U-label U1, and that U-label U1 must be capable of being produced by conversion from A-label A1. Among other things, this implies that both U-labels and A-labels must be strings in Unicode NFC [Unicode-UAX15] normalized form. These strings MUST contain only characters specified elsewhere in this document series, and only in the contexts indicated as appropriate.

Any rules or conventions that apply to DNS labels in general apply to whichever of the U-label or A-label would be more restrictive. There are two exceptions to this principle. First, the restriction to ASCII characters does not apply to the U-label. Second, expansion of the A-label form to a U-label may produce strings that are much longer than the normal 63 octet DNS limit (potentially up to 252 characters) due to the compression efficiency of the Punycode algorithm. Such extended-length U-labels are valid from the standpoint of IDNA, but caution should be exercised as shorter limits may be imposed by some applications.

For context, applications that are not IDNA-aware treat all LDH labels as valid for appearance in DNS zone files and queries and some of them may permit additional types of labels (i.e., not impose the LDH restriction). IDNA-aware applications permit only A-labels and NR-LDH labels to appear in zone files and queries. U-labels can appear, along with the other two, in presentation and user interface forms, and in protocols that use IDNA forms but that do not involve the DNS itself. 
Specifically, for IDNA-aware applications and contexts, the three allowed categories are A-label, U-label, and NR-LDH label. Of the Reserved LDH labels (R-LDH labels) only A-labels are valid for IDNA use.

Strings that appear to be A-labels or U-labels are processed in various operations of the Protocol document [RFC5891]. Those strings are not yet demonstrably conformant with the conditions outlined above because they are in the process of validation. Such strings may be referred to as "unvalidated", "putative", or "apparent", or as being "in the form of" one of the label types to indicate that they have not been verified to meet the specified conformance requirements.

Unvalidated A-labels are known only to be XN-labels, while Fake A-labels have been demonstrated to fail some of the A-label tests. Similarly, unvalidated U-labels are simply non-ASCII labels that may or may not meet the requirements for U-labels.

\subsubsection{NR-LDH Label}

These specifications use the term "NR-LDH label" strictly to refer to an all-ASCII label that obeys the LDH label syntax discussed in Section 2.3.1 and that is neither an IDN nor a label form reserved by IDNA (R-LDH label). It should be stressed that all A-labels obey the "hostname" [RFC0952] rules other than the length restriction in those rules.

\subsubsection{Internationalized Domain Name and Internationalized Label}

An "internationalized domain name" (IDN) is a domain name that contains at least one A-label or U-label, but that otherwise may contain any mixture of NR-LDH labels, A-labels, or U-labels. Just as has been the case with ASCII names, some DNS zone administrators may impose restrictions, beyond those imposed by DNS or IDNA, on the characters or strings that may be registered as labels in their zones. Because of the diversity of characters that can be used in a U-label and the confusion they might cause, such restrictions are mandatory for IDN registries and zones even though the particular restrictions are not part of these specifications (the issue is discussed in more detail in section 4.3 of the Protocol document [RFC5891]. Because these restrictions, commonly known as "registry restrictions", only affect what can be registered and not lookup processing, they have no effect on the syntax or semantics of DNS protocol messages; a query for a name that matches no records will yield the same response regardless of the reason why it is not in the zone. Clients issuing queries or interpreting responses cannot be 
assumed to have any knowledge of zone-specific restrictions or conventions. See the section on registration policy in the Rationale document [RFC5894] for additional discussion.

"Internationalized label" is used when a term is needed to refer to a single label of an IDN, i.e., one that might be any of an NR-LDH label, A-label, or U-label. There are some standardized DNS label formats, such as the "underscore labels" used for service location (SRV) records [RFC2782], that do not fall into any of the three categories and hence are not internationalized labels.

\subsubsection{Label Equivalence}

In IDNA, equivalence of labels is defined in terms of the A-labels. If the A-labels are equal in a case-independent comparison, then the labels are considered equivalent, no matter how they are represented. Because of the isomorphism of $\mathrm{A}-1$ abels and U-labels in IDNA2008, it is possible to compare U-labels directly; see the Protocol document [RFC5891] for details. Traditional LDH labels already have a notion of equivalence: within that list of characters, uppercase and lowercase are considered equivalent. The IDNA notion of equivalence is an extension of that older notion but, because the protocol does not specify any mandatory mapping and only those isomorphic forms are considered, the only equivalents are:

o Exact (bit-string identity) matches between a pair of U-labels.

- Matches between a pair of A-labels, using normal DNS case-insensitive matching rules.

- Equivalence between a U-label and an A-label determined by translating the U-label form into an A-label form and then testing for a match between the A-labels using normal DNS case-insensitive matching rules.

\subsubsection{ACE Prefix}

The "ACE prefix" is defined in this document to be a string of ASCII characters, "xn--", that appears at the beginning of every A-label. "ACE" stands for "ASCII-Compatible Encoding".

\subsubsection{Domain Name slot}

A "domain name slot" is defined in this document to be a protocol element or a function argument or a return value (and so on) explicitly designated for carrying a domain name. Examples of domain name slots include the QNAME field of a DNS query; the name argument of the gethostbyname() or getaddrinfo() standard C library functions; 
the part of an email address following the at sign ("Q") in the parameter to the SMTP MAIL or RCPT commands or the "From:" field of an email message header; and the host portion of the URI in the "src" attribute of an HTML "<IMG>" tag. A string that has the syntax of a domain name but that appears in general text is not in a domain name slot. For example, a domain name appearing in the plain text body of an email message is not occupying a domain name slot.

An "IDNA-aware domain name slot" is defined for this set of documents to be a domain name slot explicitly designated for carrying an internationalized domain name as defined in this document. The designation may be static (for example, in the specification of the protocol or interface) or dynamic (for example, as a result of negotiation in an interactive session).

Name slots that are not IDNA-aware obviously include any domain name slot whose specification predates IDNA. Note that the requirements of some protocols that use the DNS for data storage prevent the use of IDNs. For example, the format required for the underscore labels used by the service location protocol [RFC2782] precludes representation of a non-ASCII label in the DNS using A-labels because those SRV-related labels must start with underscores. Of course, non-ASCII IDN labels may be part of a domain name that also includes underscore labels.

\subsubsection{Order of Characters in Labels}

Because IDN labels may contain characters that are read, and preferentially displayed, from right to left, there is a potential ambiguity about which character in a label is "first". For the purposes of these specifications, labels are considered, and characters numbered, strictly in the order in which they appear "on the wire". That order is equivalent to the leftmost character being treated as first in a label that is read left to right and to the rightmost character being first in a label that is read right to left. The Bidi specification contains additional discussion of the conditions that influence reading order.

\subsubsection{Punycode is an Algorithm, Not a Name or Adjective}

There has been some confusion about whether a "Punycode string" does or does not include the ACE prefix and about whether it is required that such strings could have been the output of the ToASCII operation (see RFC 3490, Section 4 [RFC3490]). This specification discourages the use of the term "Punycode" to describe anything but the encoding method and algorithm of RFC 3492 [RFC3492]. The terms defined above are preferred as much more clear than the term "Punycode string". 


\section{IANA Considerations}

IANA actions for this version of IDNA (IDNA2008) are specified in the Tables document [RFC5892]. An overview of the relationships among the various IANA registries appears in the Rationale document [RFC5894]. This document does not specify any actions for IANA.

4. Security Considerations

4.1. General Issues

Security on the Internet partly relies on the DNS. Thus, any change to the characteristics of the DNS can change the security of much of the Internet.

Domain names are used by users to identify and connect to Internet hosts and other network resources. The security of the Internet is compromised if a user entering a single internationalized name is connected to different servers based on different interpretations of the internationalized domain name. In addition to characters that are permitted by IDNA2003 and its mapping conventions (see Section 4.6), the current specification changes the interpretation of a few characters that were mapped to others in the earlier version; zone administrators should be aware of the problems that this might raise and take appropriate measures. The context for this issue is discussed in more detail in the Rationale document [RFC5894].

In addition to the Security Considerations material that appears in this document, the Bidi document [RFC5893] contains a discussion of security issues specific to labels containing characters from scripts that are normally written right to left.

\subsection{U-label Lengths}

Labels associated with the DNS have traditionally been limited to 63 octets by the general restrictions in RFC 1035 and by the need to treat them as a six-bit string length followed by the string in actual calls to the DNS. That format is used in some other applications and, in general, that representations of domain names as dot-separated labels and as length-string pairs have been treated as interchangeable. Because A-labels (the form actually used in the DNS) are potentially much more compressed than UTF-8 (and UTF-8 is, in general, more compressed that UTF-16 or UTF-32), U-labels that obey all of the relevant symmetry (and other) constraints of these documents may be quite a bit longer, potentially up to 252 characters (Unicode code points). A fully-qualified domain name containing several such labels can obviously also exceed the nominal 255 octet 
limit for such names. Application authors using U-labels must exert due caution to avoid buffer overflow and truncation errors and attacks in contexts where shorter strings are expected.

\subsection{Local Character Set Issues}

When systems use local character sets other than ASCII and Unicode, these specifications leave the problem of converting between the local character set and Unicode up to the application or local system. If different applications (or different versions of one application) implement different rules for conversions among coded character sets, they could interpret the same name differently and contact different servers. This problem is not solved by security protocols, such as Transport Layer security (TLS) [RFC5246], that do not take local character sets into account.

\subsection{Visually Similar Characters}

To help prevent confusion between characters that are visually similar (sometimes called "confusables"), it is suggested that implementations provide visual indications where a domain name contains multiple scripts, especially when the scripts contain characters that are easily confused visually, such as an omicron in Greek mixed with Latin text. Such mechanisms can also be used to show when a name contains a mixture of Simplified Chinese characters with Traditional ones that have Simplified forms, or to distinguish zero and one from uppercase "O" and lowercase "L". DNS zone administrators may impose restrictions (subject to the limitations identified elsewhere in these documents) that try to minimize characters that have similar appearance or similar interpretations.

If multiple characters appear in a label and the label consists only of characters in one script, individual characters that might be confused with others if compared separately may be unambiguous and non-confusing. On the other hand, that observation makes labels containing characters from more than one script (often called "mixedscript labels") even more risky -- users will tend to see what they expect to see and context is a powerful reinforcement to perception. At the same time, while the risks associated with mixed-script labels are clear, simply prohibiting them will not eliminate problems, especially where closely related scripts are involved. For example, there are many strings that are entirely in Greek or Cyrillic scripts that can be confused with each other or with Latin script strings.

It is worth noting that there are no comprehensive technical solutions to the problems of confusable characters. One can reduce the extent of the problems in various ways, but probably never 
eliminate it. Some specific suggestions about identification and handling of confusable characters appear in a Unicode Consortium publication [Unicode-UTR36].

4.5. IDNA Lookup, Registration, and the Base DNS Specifications

The Protocol specification [RFC5891] describes procedures for registering and looking up labels that are not compatible with the preferred syntax described in the base DNS specifications (see Section 2.3.1) because they contain non-ASCII characters. These procedures depend on the use of a special ASCII-compatible encoding form that contains only characters permitted in hostnames by those earlier specifications. The encoding used is Punycode [RFC3492]. No security issues such as string length increases or new allowed values are introduced by the encoding process or the use of these encoded values, apart from those introduced by the ACE encoding itself.

Domain names (or portions of them) are sometimes compared against a set of domains to be given special treatment if a match occurs, e.g., treated as more privileged than others or blocked in some way. In such situations, it is especially important that the comparisons be done properly, as specified in the "Requirements" section of the Protocol document [RFC5891]. For labels already in ASCII form, the proper comparison reduces to the same case-insensitive ASCII comparison that has always been used for ASCII labels although IDNA-aware applications are expected to look up only A-labels and NR-LDH labels, i.e., to avoid looking up R-LDH labels that are not A-labels.

The introduction of IDNA meant that any existing labels that start with the ACE prefix would be construed as A-labels, at least until they failed one of the relevant tests, whether or not that was the intent of the zone administrator or registrant. There is no evidence that this has caused any practical problems since RFC 3490 was adopted, but the risk still exists in principle.

\subsection{Legacy IDN Label Strings}

The URI Standard [RFC3986] and a number of application specifications (e.g., SMTP [RFC5321] and HTTP [RFC2616]) do not permit non-ASCII labels in DNS names used with those protocols, i.e., only the A-label form of IDNs is permitted in those contexts. If only A-labels are used, differences in interpretation between IDNA2003 and this version arise only for characters whose interpretation have actually changed (e.g., characters, such as ZWJ and ZWNJ, that were mapped to nothing in IDNA2003 and that are considered legitimate in some contexts by these specifications). Despite that prohibition, there are a significant number of files and databases on the Internet in which 
domain name strings appear in native-character form; a subset of those strings use native-character labels that require IDNA2003 mapping to produce valid A-labels. The treatment of such labels will vary by types of applications and application-designer preference: in some situations, warnings to the user or outright rejection may be appropriate; in others, it may be preferable to attempt to apply the earlier mappings if lookup strictly conformant to these specifications fails or even to do lookups under both sets of rules. This general situation is discussed in more detail in the Rationale document [RFC5894]. However, in the absence of care by registries about how strings that could have different interpretations under IDNA2003 and the current specification are handled, it is possible that the differences could be used as a component of name-matching or name-confusion attacks. Such care is therefore appropriate.

\subsection{Security Differences from IDNA2003}

The registration and lookup models described in this set of documents change the mechanisms available for lookup applications to determine the validity of labels they encounter. In some respects, the ability to test is strengthened. For example, putative labels that contain unassigned code points will now be rejected, while IDNA2003 permitted them (see the Rationale document [RFC5894] for a discussion of the reasons for this). On the other hand, the Protocol specification no longer assumes that the application that looks up a name will be able to determine, and apply, information about the protocol version used in registration. In theory, that may increase risk since the application will be able to do less pre-lookup validation. In practice, the protection afforded by that test has been largely illusory for reasons explained in RFC 4690 [RFC4690] and elsewhere in these documents.

Any change to the Stringprep [RFC3454] procedure that is profiled and used in IDNA2003, or, more broadly, the IETF's model of the use of internationalized character strings in different protocols, creates some risk of inadvertent changes to those protocols, invalidating deployed applications or databases, and so on. But these specifications do not change Stringprep at all; they merely bypass it. Because these documents do not depend on Stringprep, the question of upgrading other protocols that do have that dependency can be left to experts on those protocols: the IDNA changes and possible upgrades to security protocols or conventions are independent issues. 


\subsection{Summary}

No mechanism involving names or identifiers alone can protect against a wide variety of security threats and attacks that are largely independent of the naming or identification system. These attacks include spoofed pages, DNS query trapping and diversion, and so on.

5. Acknowledgments

The initial version of this document was created largely by extracting text from early draft versions of the Rationale document [RFC5894]. See the section of this name and the one entitled "Contributors", in it.

Specific textual suggestions after the extraction process came from Vint Cerf, Lisa Dusseault, Bill McQuillan, Andrew Sullivan, and Ken Whistler. Other changes were made in response to more general comments, lists of concerns or specific errors from participants in the Working Group and other observers, including Lyman Chapin, James Mitchell, Subramanian Moonesamy, and Dan Winship.

6. References

6.1. Normative References

[ASCII] American National Standards Institute (formerly United States of America Standards Institute), "USA Code for Information Interchange", ANSI X3.4-1968, 1968. ANSI X3.4-1968 has been replaced by newer versions with slight modifications, but the 1968 version remains definitive for the Internet.

[RFC1034] Mockapetris, P., "Domain names - concepts and facilities", STD 13, RFC 1034, November 1987.

[RFC1035] Mockapetris, P., "Domain names - implementation and specification", STD 13, RFC 1035, November 1987.

[RFC1123] Braden, R., "Requirements for Internet Hosts Application and Support", STD 3, RFC 1123, October 1989.

[RFC2119] Bradner, S., "Key words for use in RFCs to Indicate Requirement Levels", BCP 14, RFC 2119, March 1997. 
[Unicode-UAX15]

The Unicode Consortium, "Unicode Standard Annex \#15:

Unicode Normalization Forms, Revision 31",

September 2009,

<http: / /www . unicode.org/reports/tr15/tr15-31.html>.

[Unicode52] The Unicode Consortium. The Unicode Standard, Version

5.2.0, defined by: "The Unicode Standard, Version

5.2.0", (Mountain View, CA: The Unicode Consortium, 2009. ISBN 978-1-936213-00-9).

<http://www. unicode.org/versions/Unicode5.2.0/>.

\subsection{Informative References}

[ IDNA2 $008-$ Mapping ]

Resnick, P. and P. Hoffman, "Mapping Characters in Internationalized Domain Names for Applications (IDNA)", Work in Progress, April 2010.

[RFC0952] Harrenstien, K., Stahl, M., and E. Feinler, "DoD Internet host table specification", RFC 952, October 1985 .

[RFC2181] Elz, R. and R. Bush, "Clarifications to the DNS Specification", RFC 2181, July 1997.

[RFC2616] Fielding, R., Gettys, J., Mogul, J., Frystyk, H., Masinter, L., Leach, P., and T. Berners-Lee, "Hypertext Transfer Protocol -- HTTP/1.1", RFC 2616, June 1999.

[RFC2673] Crawford, M., "Binary Labels in the Domain Name System", RFC 2673, August 1999.

[RFC2782] Gulbrandsen, A., Vixie, P., and L. Esibov, "A DNS RR for specifying the location of services (DNS SRV)", RFC 2782, February 2000.

[RFC3454] Hoffman, P. and M. Blanchet, "Preparation of Internationalized Strings ("stringprep")", RFC 3454, December 2002 .

[RFC3490] Faltstrom, P., Hoffman, P., and A. Costello, "Internationalizing Domain Names in Applications (IDNA) ", RFC 3490, March 2003.

[RFC3491] Hoffman, P. and M. Blanchet, "Nameprep: A Stringprep Profile for Internationalized Domain Names (IDN)", RFC 3491, March 2003. 
[RFC3492] Costello, A., "Punycode: A Bootstring encoding of Unicode for Internationalized Domain Names in Applications (IDNA)", RFC 3492, March 2003.

[RFC3986] Berners-Lee, T., Fielding, R., and L. Masinter, "Uniform Resource Identifier (URI): Generic Syntax", STD 66, RFC 3986, January 2005 .

[RFC4690] Klensin, J., Faltstrom, P., Karp, C., and IAB, "Review and Recommendations for Internationalized Domain Names (IDNs)", RFC 4690, September 2006.

[RFC5246] Dierks, T. and E. Rescorla, "The Transport Layer Security (TLS) Protocol Version 1.2", RFC 5246, August 2008 .

[RFC5321] Klensin, J., "Simple Mail Transfer Protocol", RFC 5321, October 2008 .

[RFC5891] Klensin, J., "Internationalized Domain Names in Applications (IDNA): Protocol", RFC 5891, August 2010.

[RFC5892] Faltstrom, P., Ed., "The Unicode Code Points and Internationalized Domain Names for Applications (IDNA)", RFC 5892, August 2010 .

[RFC5893] Alvestrand, H. and C. Karp, "Right-to-Left Scripts for Internationalized Domain Names for Applications (IDNA)", RFC 5893, August 2010.

[RFC5894] Klensin, J., "Internationalized Domain Names for Applications (IDNA): Background, Explanation, and Rationale", RFC 5894, August 2010.

[Unicode-UTR36]

The Unicode Consortium, "Unicode Technical Report \#36: Unicode Security Considerations, Revision 7", July 2008, <http: //www . unicode.org/reports/tr36/tr36-7.html>. 


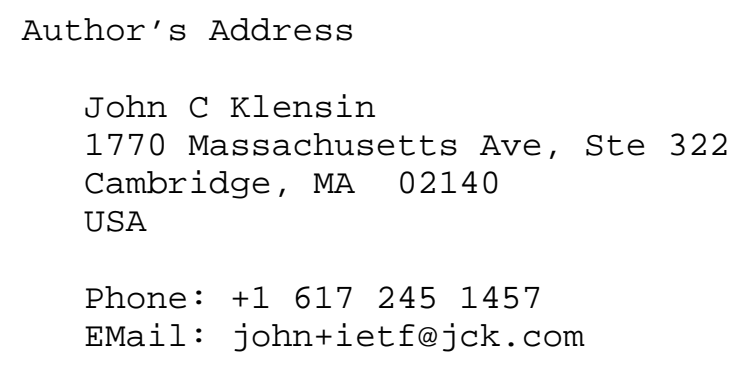

